\title{
Chemotherapie bis zum letzten Atemzug?
}

Ergebnisse einer Studie zeigen, dass von 1.231 Patienten mit Lungen- oder Kolorektalkrebs im Stadium IV 16\% in den 14 Tagen vor ihrem Tod noch eine Chemotherapie bekamen, $9 \%$ in den letzten 30 Tagen noch auf der Intensivstation und $40 \%$ auf einer anderen Station behandelt wurden. Fast 50\% der Patienten erhielten mindestens eine dieser Maßnahmen. Bei Patienten, mit denen ein Arzt mehr als 30 Tage vor dem Tod über die medizinische Versorgung am Lebensende gesprochen hatte, erfolgten Chemotherapien sowie Aufenthalte auf Intensiv- und Normalstation auch unter Berücksichtigung von Alter und Überlebenszeit - signifikant seltener als bei Patienten, deren letzte Wünsche später oder gar nicht erfragt worden waren. Zudem verbrachten sie die letzte Le- bensphase häufiger und früher in einem Hospiz. Nur 88\% der Studienteilnehmer - alle hatten noch weniger als 15 Monate zu leben - sprachen irgendwann mit einem Arzt über lebenserhaltende Maßnahmen, Palliativversorgung oder Hospizunterbringung. Aus den Krankenakten ging hervor, dass 39\% der Gespräche erst in den letzten 30 Tagen vor dem Tod stattfanden, $63 \%$ der Patienten wurden zu diesem Zeitpunkt stationär behandelt. Die Anwesenheit eines Onkologen bei diesem Gespräch erhöhte die Wahrscheinlichkeit für eine Chemotherapie in den letzten zwei Lebenswochen. „Früher mit den Patienten über das Lebensende zu sprechen, könnte die medizinische Versorgung bei fortgeschrittenen Krebserkrankungen ändern und dazu beitragen, dass sie stärker dem

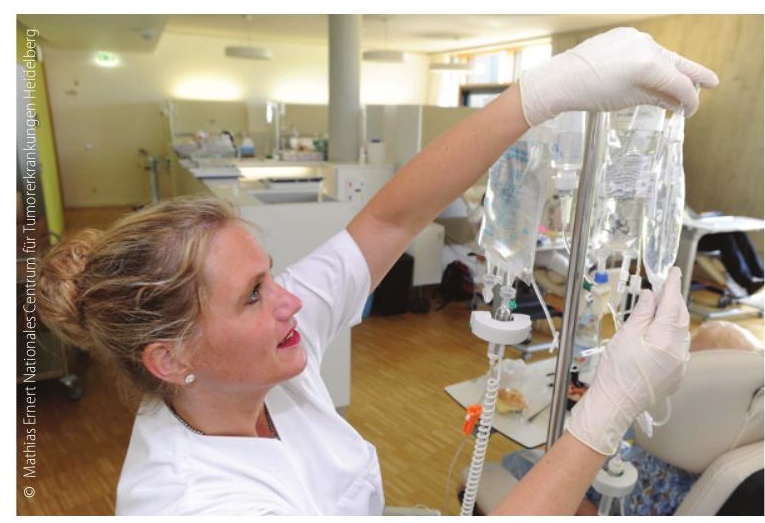

Häufig wird noch bis kurz vor dem Tod eines Patienten chemotherapiert.

Willen der Patienten entspricht“, resümieren die Studienautoren. Beate Schumacher

Mack JW et al. Associations Between End-of-Life Discussion Characteristics and Care Received Near Death: A Prospective Cohort Study. J Clin Oncol. 2012 Nov 13. [Epub ahead of print]

\section{Antivirale Therapie zum Schutz vor Rezidiv?}

In einer taiwanischen Studie wurde eine tertiärpräventive Wirkung von Nukleosidanaloga gegen ein Rezidiv eines resezierten HBV-assoziierten hepatozellulären Karzinoms (HCC) beschrieben. Es waren Versicherungsdaten der gesamten Bevölkerung aus den Jahren 2003 bis 2010 ausgewertet worden. In dieser Zeit hatten 4.569 Hepatitis-B-Patienten wegen eines HCC eine Leberresektion erhalten. 518 waren danach mit (mindestens) einem Nukleosidanalogon - Lamivudin, Entecavir oder Telbivudin - behandelt worden. Bei den behandelten Patienten bestand häufiger als bei den unbehandelten Patienten eine Leberzirrhose (48,6 vs. 38,7\%). HCC-Rezidive wurden in der mittleren Nachbeobachtungszeit von gut zwei Jahren bei 20,5\% der Patienten mit und 43,6\% der Patienten ohne antivirale Therapie diagnostiziert. Berücksichtigte man, dass einige Patienten starben, bevor sich ein Rezidiv entwickeln konnte, ergab sich für die Therapiegruppe eine signifikant niedrigere 6-Jahres-Rezidivrate als in der Vergleichsgruppe (45,6 vs. 54,6\%). Demnach müssen zwölf Patienten mit Nukleosidanaloga behandelt werden, um einen Rezi- divtumor zu verhindern. Unter Berücksichtigung anderer Rezidiv-Risikofaktoren, v.a. der höheren Zirrhoserate in der Therapiegruppe, war bei den antiviral behandelten Patienten das Rezidivrisiko um $27 \%$ reduziert.

Die 6-Jahres-Mortalität war mit 29,0\% im Vergleich zu 42,4\% ohne antivirale Behandlung signifikant geringer. Um einen Todesfall in sechs Jahren zu verhindern, müssten acht Patienten ein Nukleosidanalogon erhalten.

Ob ein kausaler Zusammenhang zwischen der Medikation und dem erniedrigten Rezidivrisiko bestehe, lasse sich aber laut den Studienautoren nicht sagen. Anna F.S. Lok, Ann Arbor, MI/USA, äußert sich in ihrem begleitenden Editorial skeptisch: Es sei eigentlich nicht zu erwarten, dass eine antivirale Therapie frühe HCC-Rezidive verhindern könne, da diese hauptsächlich durch nicht entdeckte Metastasen verursacht würden. Beate Schumacher

Wu C-Y et al. Association Between Nucleoside Analogues and Risk of Hepatitis B Virus-Related Hepatocellular Carcinoma Recurrence Following Liver Resection. JAMA. 2012;308(18):1906-14.

\section{kurz notiert}

\section{Forschungsstandort Deutschland}

Nebennierenkarzinome gezielt bekämpfen +++ Martin Fassnacht, München, erhielt für seine Forschung zur Chemotherapie bei Nebennierenkarzinomen den Wissenschaftspreis der Arbeitsgemeinschaft internistische Onkologie (AIO). Nach der internationalen, multizentrischen Studie reduziert die Viererkombination Mitotane, Etoposid, Doxorubicin und Cisplatin deutlich effektiver als die Kombination Mitotane und Streptozotocin die Tumormasse. Auch das 1-Jahres-Überleben ist unter der Viererkombination besser. [Fassnacht M et al. FIRM-ACT Study Group. N Engl J Med. 2012;366(23):2189-97].

Neuer Therapieansatz gefunden? +++ Eine spezifische Histon-Deacetylase (HDAC), HDAC11, wurde als vielversprechende Zielstruktur für neue Krebstherapien identifiziert. Forscher des Deutschen Krebsforschungszentrums (DKFZ) fanden heraus, dass u. a. Brust-, Leber- oder Nierenkrebszellen im Vergleich zu gesunden Zellen besonders viel HDAC11 produzieren. Eine Hemmung der HDAC11-Produktion in Zelllinien verschiedener Krebs- und gesunder Zellen reduzierte die Leistungsfähigkeit und steigerte die Apoptose der Krebszellen, ohne die gesunden Zellen zu beeinflussen. Eine selektive HADC11-Blockade könnte demnach nur die Krebszellen treffen [Deubzer HE et al. Int J Cancer. 2012 Oct 1. (Epub ahead of print)] 